\title{
Paratesticular Embryonal Rhabdomyosarcoma with Lung Metastasis - A Rare Case Report
}

\author{
Anusha $\mathbf{G}^{1}$, Thanka $\mathbf{J}^{1}$, Lawrence D’Cruze ${ }^{1 *}$, Barathi $\mathbf{G}^{1}$, Natarajan $\mathbf{K}^{1}$ and Julius Xavier Scott ${ }^{2}$ \\ 'Department of Pathology, Sri Ramachandra Institute of Higher Education and Research, Chennai, Tamil Nadu, India \\ ${ }^{2}$ Department of Paediatric Hemato-oncology,Sri Ramachandra Institute of Higher Education and Research, Chennai, Tamil Nadu, India.
}

\begin{abstract}
Paratesticular rhabdomyosarcomas (RMS) are very rare malignant tumors arising from the mesenchymal tissues of tunica, epididymis or spermatic cord. They present as painless hard masses in inguinoscrotal region, and large tumors can be mistaken for testicular tumors. They can spread to retroperitoneal lymph nodes or hematogenously metastasize to lung, bones and bone marrow. Here, we report a case of Embryonal RMS with spindle cell component presenting with painless scrotal mass and lung metastasis at initial diagnosis.
\end{abstract}

Keywords: Paratesticular RMS, Embryonal, Lung Metastasis, Myogenin

\section{Introduction}

Rhabdomyosarcomas (RMS) of the paratesticular region are very rare and comprise only $7 \%$ of all RMS and $17 \%$ of all intrascrotal malignant tumors (children $<15$ years old). ${ }^{(1)}$ They are aggressive tumors and present as painless hard scrotal masses. ${ }^{(2)}$ Embryonal subtype is the most common histomorphology encountered. ${ }^{(3)}$ Diagnosis is based on a high degree of suspicion, radio-imaging for staging the tumor and histological examination \& immunohistochemical confirmation. Multi-modality treatment comprising of high inguinal orchidectomy, systemic chemotherapy and radiotherapy is helpful in attaining maximum tumor control. ${ }^{(4)}$

\section{Case Report}

A 5-year-old male child was brought by his mother with the complaints of right testicular swelling for 8 months duration. The swelling was gradually progressive and it was not associated with pain or fever. On palpation of right scrotal swelling, there was no warmth or tenderness and the swelling was firm in consistency. Left testis was unremarkable upon examination.

Ultrasound of scrotum showed suspiciously elongated right testis with a large solid isoechoic heterogenous mass with internal vascularity. The provisional diagnosis was right testicular tumor, following which a PET-CT scan was performed which revealed a heterogenous lesion (134 x 95 x $92 \mathrm{~mm}$ ) in the right scrotum replacing the testis, with ${ }^{18} \mathrm{~F}$-fluoro-2-deoxy-D-glucose (FDG) avid solid and cystic components noted within. Also, 2 nodules of size $4 \times 4 \mathrm{~mm}$ and $2 \times 2 \mathrm{~mm}$ were noted in the postero-basal segment and apical segment of right lower and upper lobes of the lung respectively.

Right orchidectomy was done. On gross examination, the external surface was grey-white and irregular. Cut surface showed a solid grey-white mass measuring $12.8 \times 7.5 \times$ $7 \mathrm{~cm}$ replacing the entire sac wall. Compressed testicular tissue was also identified. Histopathological examination of orchidectomy specimen showed sheets of small round to oval cells with scant cytoplasm, hyperchromatic nuclei with focal anaplasia and areas of spindled out cells. Extensive rhabdomyoblastic differentiation was made out in some areas. Circumferential margin was focally involved. By IHC, the tumor cells were positive for Vimentin, Desmin, Myogenin and TLE1 (10\%) focally in spindle cells. The diagnosis was Paratesticular Embryonal rhabdomyosarcoma with extensive rhabdomyoblastic differentiation, focal spindle cell component and diffuse anaplasia.

Post surgically, patient was placed on systemic chemotherapy VAC regimen (Vincristine, Dactinomycin and Cyclophosphamide). After 6 cycles of chemotherapy, PET-CT scan was repeated to assess metastasis. The lung nodules were showing no increase in size to the previous scan and there was no active metabolic residual disease in scrotal sac. Following this, Right hemiscrotectomy along with biopsy of lung nodule was done. The right hemiscrotectomy tissue specimen showed granulation tissue formation, while the lung biopsy confirmed the presence of metastatic tumor deposits. Patient is currently on chemotherapy with routine follow-up. 


\section{Discussion}

Rhabdomyosarcoma (RMS) is one of the commonest soft tissue sarcomas of childhood. However, paratesticular location of RMS is very rare and constitutes only $7 \%$ of all rhabdomyosarcomas. They develop from the mesenchymal tissues of tunica, epididymis or spermatic cord. ${ }^{(5)}$ The

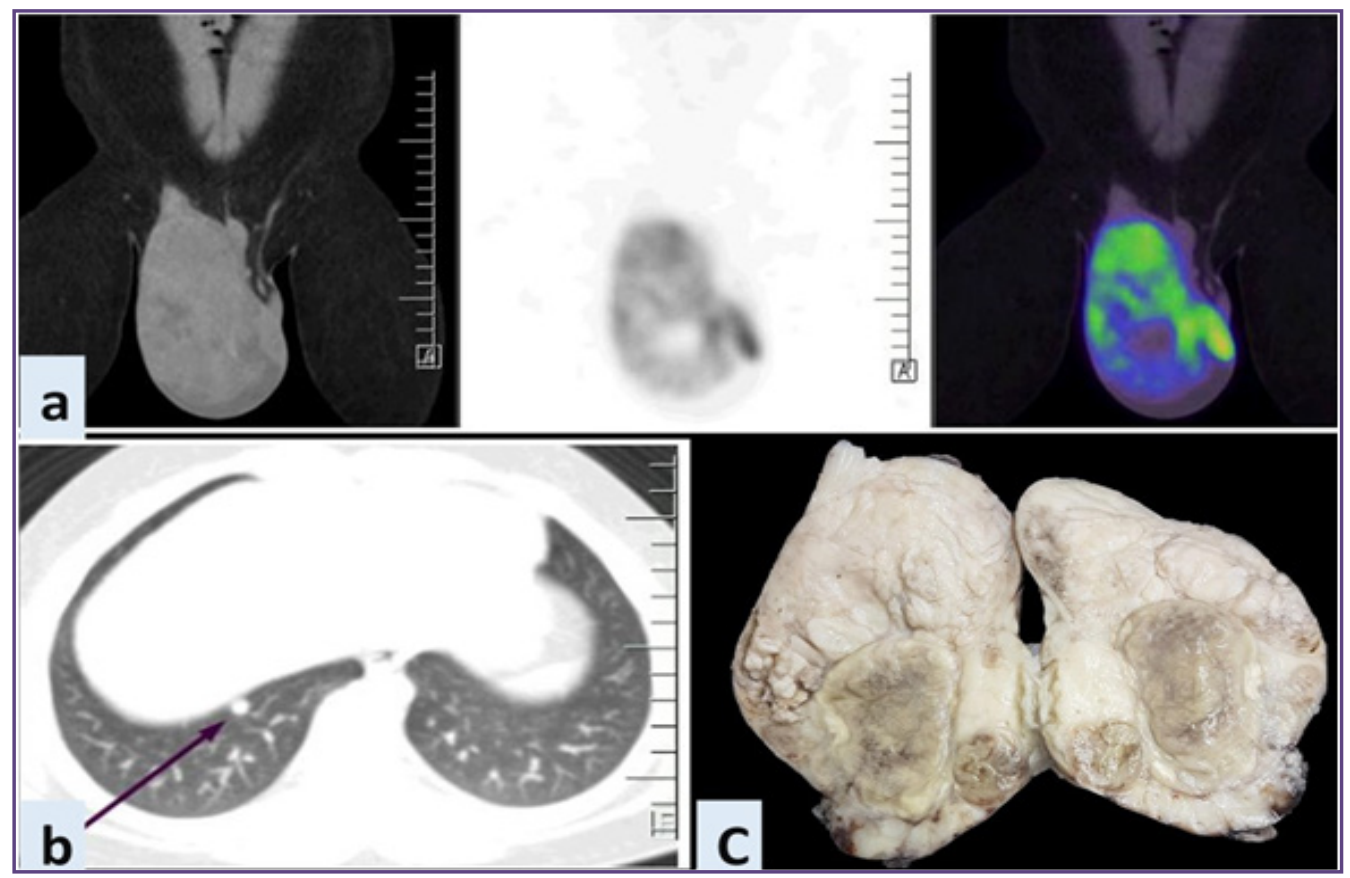

Fig. 1a: PET CT scan showing intrascrotal tumor with FDG uptake. b.) Lung nodule seen in the right lower lobe. C.) Gross specimen of right orchidectomy showing grey-white solid tumor with compressed testicular tissue.

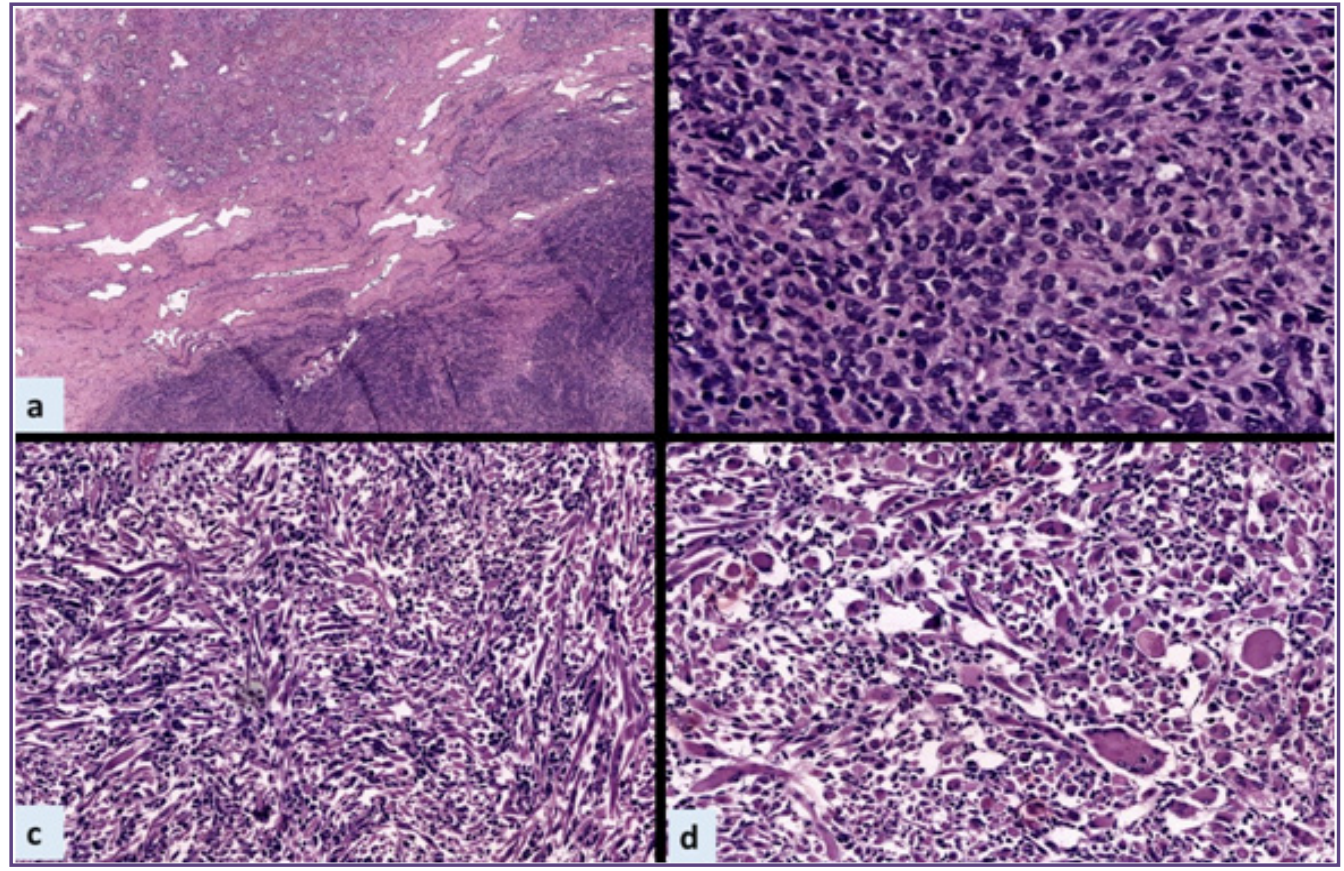

Fig. 2a: Tumor with adjacent compressed testicular parenchyma b.) Embryonal RMS showing sheets of small round cells c.) Spindle cell component d.) Rhabdomyoblasts (strap cells). 


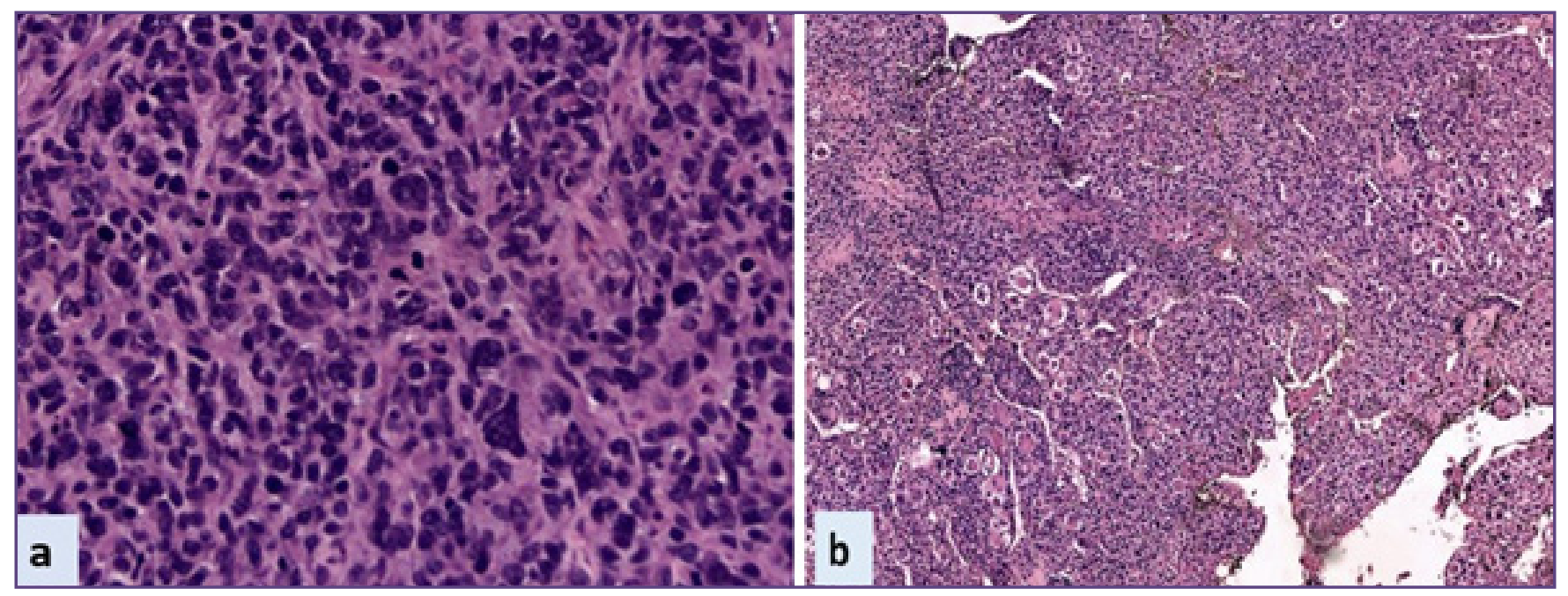

Fig. 3a: Scattered large anaplastic cells b.) Lung nodule showing tumor deposit.

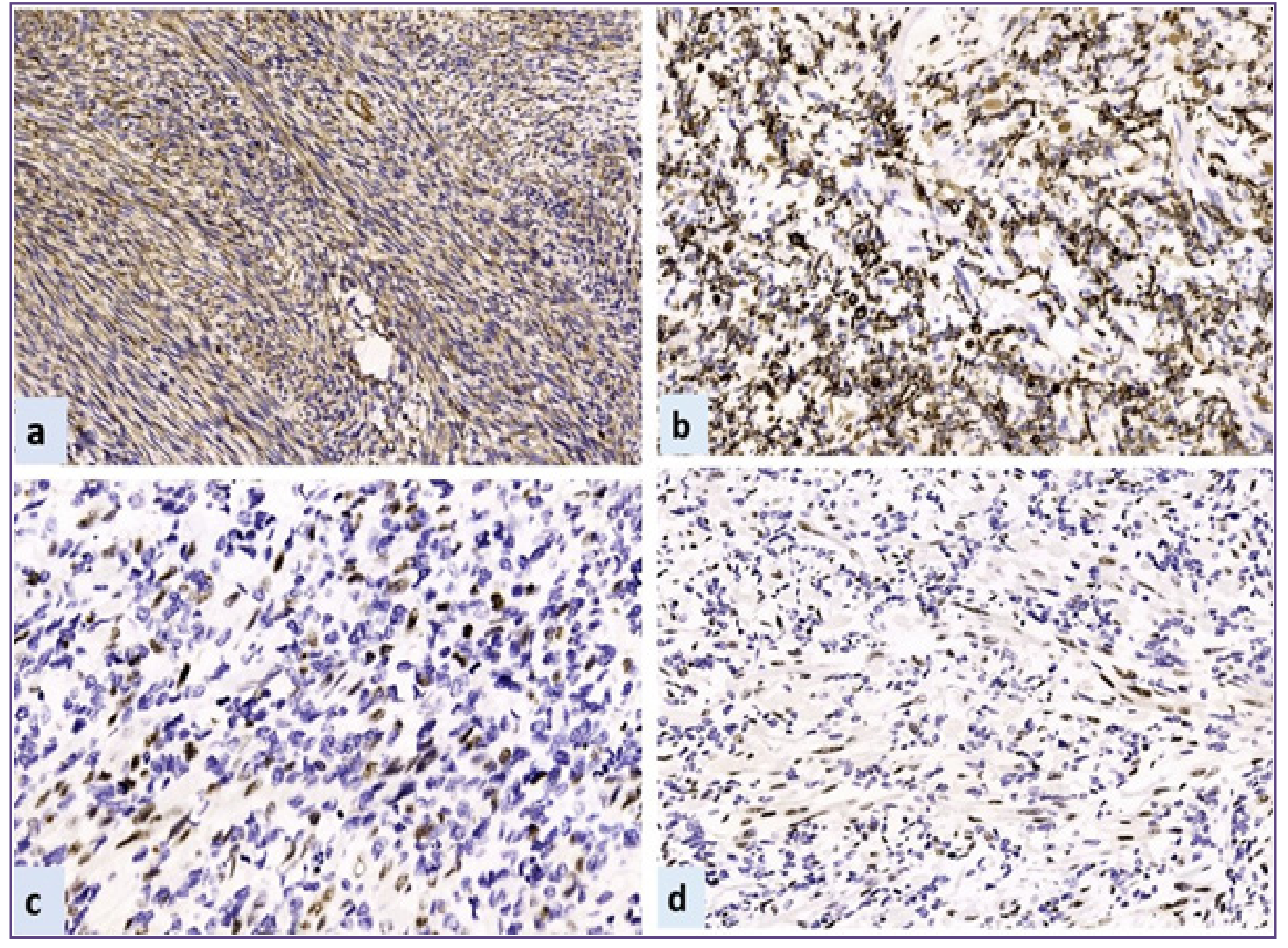

Fig. 4a.: Tumor cells showing positivity for Vimentin b.) Desmin c.) Myogenin d.) Focally for TLE1. 
other common sarcomas in the paratesticular region are leiomyosarcoma (32\%), liposarcoma (20\%), and malignant fibrous histiocytoma $(13 \%)$. ${ }^{(6)}$ There are two peaks of incidence, with the first peak more commonly in 1-5 years followed by a second peak in adolescence. ${ }^{(2)(5)(7)}$

The tumor presents as a painless hard mass in the inguinoscrotal region which rarely invades the scrotal skin. The tumor can be confused with conditions like epididymoorchitis, testicular torsion, scrotal abscess, hydrocoele , strangulated hernia and rarely testicular tuberculosis. ${ }^{(2)(5)}$

However, painful lesions are seen in only $7 \%$ of cases. (6) Large tumors can be mistaken for tumors of testicular origin as in our case. Spread of tumor can occur via lymphatics preferentially to retroperitoneal lymph nodes and by hematogenous spread to lung, bone and bone marrow. ${ }^{(4)(5)(8)}$

For initial workup, scrotal ultrasonography is the imaging modality of choice. A frequency of $7.5-10 \mathrm{MHz}$ is required to distinguish testicular and paratesticular lesions. USG helps to demonstrate the solid component, and eliminate differential diagnosis like hydrocoele and varicocoele. CT scan of thoraco-abdomino-pelvic regions is performed to look for retroperitoneal and mediastinal lymphadenopathy and distant metastasis. ${ }^{18} \mathrm{~F}$-fluoro-2deoxy-D-glucose(FDG) PET-CT scan has been shown to be a sensitive tool in staging and re-staging of RMS and also in assessment of chemotherapy response. ${ }^{(7)}$

Based on histomorphology, three types of RMS have been identified, namely embryonal type, alveolar type and pleomorphic type, of which embryonal type is the most common subtype, making up approximately $80 \%$ of the cases. Additional variants have more recently been identified, such as spindle cell RMS and sclerosing RMS that comprise $5 \%$ to $10 \%$ of RMS. ${ }^{(7)}$ Rhabdomyoblast is the characteristic cell but is not essential for diagnosis. (2)(4) In embryonal RMS, small round cells with scant rim of eosinophilic cytoplasm and small oval nuclei as well as spindled out cells with cross striations are seen in a loose myxoid stroma. Occasional tumor cells with abundant eosinophilic cytoplasm (strap cells) are a feature of rhabdomyoblastic differentiation. On Immunohistochemistry, the tumour cells are immunoreactive for desmin, actin and myogenin and negative for Pan CK, S100 and CD99. Immunoreactivity for myogenin clinches the diagnosis. ${ }^{(4)}$

The differential diagnosis (DD) for rhabdomyosarcoma include leiomyosarcoma, fibrosarcoma, dedifferentiated liposarcoma, malignant fibrous histiocytoma, malignant triton tumor and malignant mesothelioma. ${ }^{(4)(9)(10)}$ However, these tumors occur more commonly in adults. Small round cell tumors should be included in the differential diagnosis of Embryonal RMS. Non Hodgkin lymphoma (NHL) of B cell type involving the testis can mimic Embryonal RMS. NHL (B cell type) will usually be positive for CD45 and CD20 and negative for desmin and myogenin. ${ }^{(11)}$

Other DD include desmoplastic small round cell tumor (DSRCT) and extraskeletal Ewing sarcoma. DSRCT mimics Embryonal RMS as it frequently shows desmin positivity and an alveolar pattern imparted by dense sclerosis that separates tumor nests cells. However, Myogenin will be negative in DSRCT and helps in differentiating the two. CD99, often used as a marker in Ewing sarcoma may be positive in rhabdomyosarcoma, whereas FLI-1 will be negative. ${ }^{(9)}$ Myogenin positivity helps to clinch the diagnosis of RMS in such cases.

Localized tumors are associated with good prognosis whereas metastatic disease is associated with poor prognosis. Multimodal therapy using systemic chemotherapy in conjunction with either surgery, radiotherapy is used to maximize tumor control. For localized tumor, high inguinal orchidectomy is the treatment of choice. Hemiscrotectomy is performed if there is scrotal violation. ${ }^{(12)}$

The use of retroperitoneal lymph node dissection (RPLND) is controversial. The protocol for Children's Oncology Group (COG) however recommends that staging ipsilateral RPLND be done for all boys aged $>10$ years, and in those $<10$ years it is reserved for those with suspicious lymph nodes on CT scan. RMS is chemo-sensitive and the most commonly used chemotherapeutic regimen is Vincristine, Dactinomycin and Cyclophosphamide (VAC). ${ }^{(7)}$

\section{Conclusion}

Paratesticular RMS are rare aggressive tumors. Due to varied presentation and histomorphology, IHC plays a crucial role in diagnosis of this tumor. Positive myogenin clinches the diagnosis. Radio-imaging plays a crucial role in diagnosis and staging.

\section{Acknowledgements}

Authors are thankful to Mr. Venkatraman for doing immunohistochemistry in this case.

\section{Funding}

None.

\section{Competing Interests}

Authors have no competing interests to declare. 


\section{References}

1. Resim S, Okur N, Bakaris S, Kilic AO, Altunoluk B. Paratesticular embryonal rhabdomyosarcoma; report of a case. Iran J Pediatr. 2009;19(4):430-4.

2. Ahmed Amine Bouchikhi, Soufiane Mellas, Mohammed Fadl Tazi, Karim Lahlaidi YK, Khadija Benhayoune, Rajae Kanab, Jalal Eddine Elammari, Abdelhak Khallouk MJEF and MHF. Embryonic paratesticular rhabdomyosarcoma: a A case report. J Med Case Rep. 2013;7(1):93.

3. Ferrari A, Bisogno G, Casanova M, Meazza C, Piva L, Cecchetto G, et al. Paratesticular rhabdomyosarcoma: Report from the Italian and German Cooperative Group. J Clin Oncol. 2002;20(2):449-55.

4. Navin Kumar Sinha. Paratesticular Rhabdomyosarcoma in Young Adult- A Case Report. J Clin Diagnostic Res. 2008;113(11):3242-7.

5. Ankit Shukla, Ramesh Bharti, Sanjeev Sharma, Rajesh Chaudhry VV. Rare case of paratesticularrhabdomyosarcoma. Pediatr Urol Case Reports. 2015;2(6):9-14.

6. Boulma R, Gargouri MM, Sallemi A, ChlifM, Fitouri Z, Kallel Y, et al. Paratesticular Pleomorphic Rhabdomyosarcoma: A Report of Two Cases. Case Rep Urol. 2013;2013:1-4.

7. Dangle PP, Correa A, Tennyson L, Gayed B, Reyes-
Múgica M, Ost M. Current management of paratesticular rhabdomyosarcoma. Urol Oncol Semin Orig Investig. 2016;34(2):84-92.

8. Kumar R, Kapoor R, Khosla D, Kumar NK, Ghoshal S, Mandal AK, et al. Paratesticular rhabdomyosarcoma in young adults: A tertiary care institute experience. Indian J Urol. 2013;29(2):110-3.

9. Parham DM, Ellison DA. Rhabdomyosarcomas in adults and children: An update. Arch Pathol Lab Med. 2006;130(10):1454-65.

10. Robert Anthony Keenan, Aisling U. Nic An Riogh, Andrea Stroiescu, Adrian Fuentes, Joan Heneghan IMC and PJD. Paratesticular sarcomas: a case series and literature review. Ther Adv Urol. 2019;11:1-8.

11. Al-Abbadi MA, Hattab EM, Tarawneh M, Orazi A, Ulbright TM. Primary testicular and paratesticular lymphoma: A retrospective clinicopathologic study of 34 cases with emphasis on differential diagnosis. Arch Pathol Lab Med. 2007;131(7):1040-6.

12. Hammond WJ, Farber BA, Price AP, Wolden SL, Heaton TE, Wexler LH, et al. Paratesticular rhabdomyosarcoma: Importance of initial therapy. J Pediatr Surg. 2017;52(2):304-8.

*Corresponding author:

Dr. Lawrence D'Cruze, Sri Ramachandra Institute of Higher Education and Research, Porur, Chennai- 600116

Phone: +91 9940265024

Email:drlawcruz@gmail.com

Date of Submission : 06/08/2019

Date of Acceptance : 20/10/2020

Financial or other Competing Interests: None.

Date of Publication : 30/11/2020 